\title{
Research on Computer Network Optimization Model Based on Neural Network Algorithm
}

\author{
Wang Yanhui \\ Department of Computer and Information Engineering, Baoding Vocational and Technical College, \\ Baoding, 071000, China \\ 251208123@qq.com
}

Keywords: Neural network algorithm; Computer network; Modern optimization methods

\begin{abstract}
The rapid development of computer networks is urgently need to improve and optimize the overall performance of the network. With the characteristics of large scale parallel processing and distributed information storage, Hopfield neural network has a unique advantage of associative memory and optimization. Optimization model of neural network algorithm-based computer network, it is the combination of the Hopfield neural Network theory and reality computer networks, modern optimization methods.
\end{abstract}

\section{Introduction}

Computer network, especially Internet, as one of the most important achievements of human technological progress in the last twenty years. It plays an important role in education, science and human life. At present, the hub server-centric network system provide the reality of the interconnection network. But it has also become a bottleneck and constraints of the network. The huge mass of information enrich the content of network, but the development of the images, sounds and other multimedia technologies make the current network operation powerlessly. Without the authority of the tower of gold, the headless network internet is easy to expand, but the more growth, the more bloated and fragile exposed.therefore.Improving the performance of the overall optimization of the network has become an inevitable result of the network development at a certain stage [1,2].

Neural network is a kind of intelligent system. It is a kind of simplification and abstraction of a kind of information processing mode of the human brain. The main features are: large scale parallel processing and distributed information storage, good adaptability, self-organization and strong learning function, association and fault tolerance function. At present, the neural network has made gratifying achievements in image processing, pattern recognition, speech synthesis and intelligent robot. It is the most challenging and fascinating research hot spot in the world. especially in the Hopfield neural network. Associated memory and optimization have shown a unique advantage from which it has just been born. It has only used 900 neural networks giving the optimal solution to the NP problem of a 30 city traveling salesman obtained with 0.2 seconds [3,4].

In order to explore the intelligent methods to solve the problems of the complexity and large-scale research train of thought, the research of computer network optimization model based on the neural network algorithm is the combination of practical computer network and the theory of Hopfield neural network [5].

\section{Hopfield Neural Network}

Currently used for network optimization is mainly Hopfield neural network, which is firstly mentioned out by an American physicist who named JJ.Hopfield in 1982. It is mainly used to simulate the memory mechanism of neural networks. It is a fully connected neural network. Fig. 1 shows the basic work principle of neurons. 


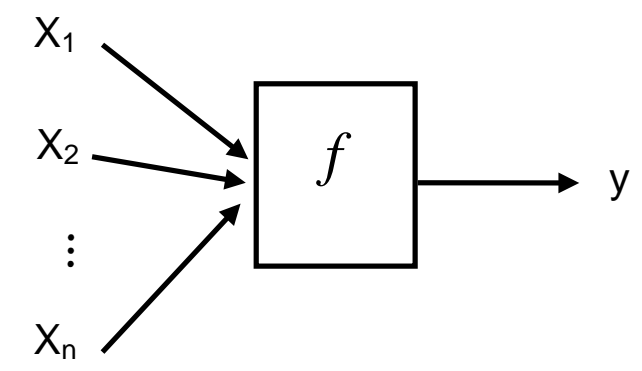

Fig. 1 The neuron model

Where $x_{i}$ and $y$ are respectively the input and output. $w_{i}$ is weight coefficient. $f$ is characteristic function, which reflects the mapping relationship between inputs and outputs, and it is usually a nonlinear function.

Neural network consists of input layer, hidden layer and output layer, which is shown in Fig. 2.

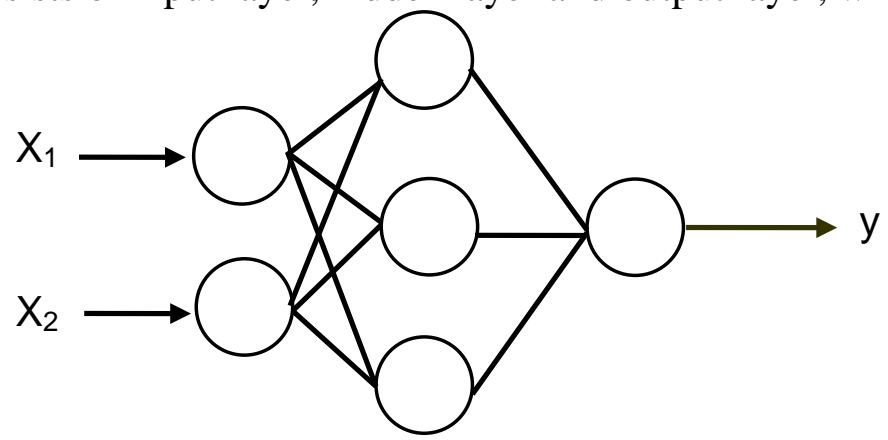

Fig. 2 The connection model of neural network

The optimization steps of Hopfield neural network are show in Fig.3.

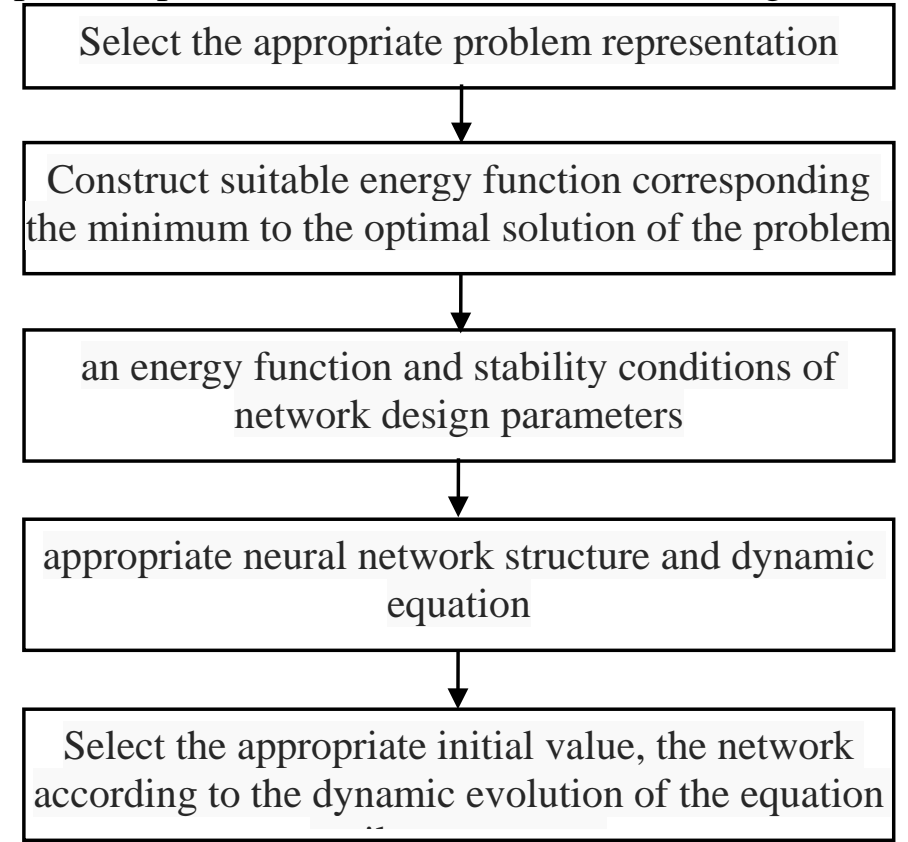

Fig.3 The steps of optimization

For each neuron, their own output signal is then fed back to itself. So it is a feedback neural network.

Optimization Mechanism. Hopfield neural network evolution is a nonlinear dynamical system. The stability of the system can be used so-called "energy function" (Ie Lyapunov or Hamiltonian) for analysis. If the energy function is considered as the objective function of an optimization problem, then from the initial state toward the stable point of the evolution process is a solution of the optimization problem. Thus, the Hopfield network evolution is a process of calculating associative memory or solving optimization problems. 
Optimization Model. Feedback network is used for two problems which are the dual. The two problems are optimization calculation and associative memory. Make W known for optimization calculation, and the purpose is to find $E$ minimum stable state. Make associative memory in a steady state which is given to find suitable $w$. Therefore, in practice, the key is how to express the objective function of the problem into the following Quadratic form of energy function:

$$
E=-\frac{1}{2} \sum_{i=1}^{N} \sum_{j=1}^{N} T_{i j} v_{i} v_{j}-\sum_{i=1}^{N} \theta_{i} v_{i}=-\frac{1}{2} x^{T} W x-x^{T} I
$$

Where $I$ is an external input vector.

Commonly used Hopfield network has two categories: discrete (DHNN) and continuous (CHNN).

Continuous network, the dynamic equation is:

$$
\left\{\begin{array}{c}
\frac{d u_{i}}{d t}=f_{i}\left(v_{1}, v_{2}, \cdots, v_{N}\right) \\
v_{i}=g_{i}\left(u_{i}\right), i=1,2, \cdots, N
\end{array}\right.
$$

Where $g_{i}$ is the commonly used sigmoid functions, $v_{i}=g_{i}\left(u_{i}\right)=\frac{1}{2}\left[1+\tanh \frac{u_{i}}{u_{0}}\right]$, controllable functions slope $u_{0}$, when $u_{0} \rightarrow 0, g_{i}$ becomes a step function.

Discrete Network, $\left\{\begin{array}{c}u_{i}=f_{i}\left(v_{1}, v_{2}, \cdots v_{N}\right) \\ v_{i}=g\left(u_{i}\right), v_{i} \in\{0,1\}, i=1,2, \cdots, N\end{array} \quad\right.$ where $\quad g_{i} \quad$ is $\quad$ a step function, $v_{i}=g\left(u_{i}\right)\left\{\begin{array}{l}1, u_{i} \succ 0 \\ 0, u_{i} \prec 0\end{array}\right.$, where $N$ is the number of neurons, $u_{i}$ and $v_{i}$ denote the i-th neuron inputs and outputs.

\section{Network flow optimization model based on neural network}

The key of network flow optimization is division of Network Flow diagram, the minimum cutting and the maximum flow problem [1].

Division Of Network Flow Diagram. It is that divide Network Flow diagram into K parts, so that the number of nodes in each part is approximately equal. Then the energy function is:

$$
E=\frac{1}{2} \sum_{i, j} \sum_{a \neq b} w_{i j} v_{i a} v_{i b}+\frac{A}{2} \sum_{a \neq b} v_{i a} v_{i b}+\frac{B}{2} \sum_{a}\left(\sum_{i} v_{i a}-\left[\frac{N}{K}\right]^{2}\right)
$$

Where unit $v_{i a}$ take 0 or $1, \mathrm{a}=1,2, \ldots \mathrm{K}, v_{i a}=1$ express node i belongs to the set of a, otherwise $v_{i a}=0$.

Minimum Cutting Problem. The minimum cutting problem is to find a kind of cutting, it make the capacity of the cut set is the smallest. A cut figure refers to a division $N \rightarrow n_{1} \cup n_{2}$. A cut set is a set of arcs (i, j), which $i \in n_{1}, j \in n_{2}$ Capacity of the cut set is the sum of the weights of its arc. Let $\mathrm{n}=(\mathrm{W}, \mathrm{t})$ as a network and $\mathrm{T}=0$. then the state of minimum energy demand equivalent to finding the $\mathrm{N}$ in the minimum cut of division of Network Flow diagram. Energy function of $\mathrm{N}$ can be written as:

$$
E=-\sum_{i=1}^{n} \sum_{j=1}^{n} w_{i j} v_{i} v_{j}
$$

Maximum Flow Problem. Given a directed graph G (V, e). There is a starting point $S$, an end point $Z$. each side of the capacity is $c_{i j}$. For each side there is a non negative $f_{i j}$ and meet: On each side have $f_{i j} \leq c_{i j}$ ( $f_{i j}=c_{i j}$ when the side saturation); Except the point $S$ and $Z$, it meet $\sum_{j} f_{i j}=\sum_{k} f_{k i}$. The point $\mathrm{S}$ and $\mathrm{Z}$ meet $\sum f_{s i}=\sum f_{i z}=W$.Have the following conclusions: It 
is concluded that the maximum value of $W$ is less than the capacity of any cut. Therefore, solving the problem of the maximum value of the network flow is equal to solving the problem of the minimum value of its cutting.

\section{Dynamic route selection model based on Neural Network}

At present the physical network of communication network is a P2P connection, you can use an undirected graph $G=(v, e)$ to express. The vertice present switching nodes and edges represent pathways [6]. Each side has a maximum capacity. In order to realize the communication between all point to point in the network, we need according to user's call and arrange routing network traffic situation. Now we commonly use the static method in accordance with the pre-arranged routing table to make choice. Due to the volume of business changes, people want to choose the dynamic routing under the circumstances in order to improve the efficiency of the neural network. The parallel computing of neural network provides the possibility for the dynamic selection of the route. Corresponding to its optimization model flowing:

Set up the graph of the network $G=(v, e)$, and make a serial number for each side of the network. 1 said path through the edge, with 0 said no. In this way, a route is expressed by 0 and 1 , $L \times M$ neurons express $L$ need $L \times M$ routes, $M$ is an alternative route number, communication network has $\mathrm{N}$ nodes [7]. Then:

The objective function:

$$
E=E_{1}+E_{2}+E_{3}
$$

Constraints conditions:

(1) Each call has one route at most.

$$
E_{1}=\frac{A}{2} \sum_{i=1}^{L} \sum_{j=1}^{M} \sum_{q=1, q \neq j}^{M} v_{i j} v_{i q}=0
$$

(2) There is a possible route for each call.

$$
E_{2}=\frac{B}{2} \sum_{i=1}^{L}\left[1-\sum_{j=1}^{M} v_{i j}\right]^{2}=0
$$

(3)The cost of selected route is the lowest.

$$
E_{3}=\frac{C}{2} \sum_{i=1}^{L} \sum_{j=1}^{M} \sum_{p=1}^{J} v_{i j} v_{p i} \sum_{k=1}^{N} C_{k}\left(l_{i j k} \vee l_{p j k}\right)
$$

\section{Cells highly competitive model based on neural network}

Computer networks, such as ATM, etc. If two or more cells destined for the same route at the same time, it will definitely involve competition. Therefore, how to set up a queue on the switch to achieve its reasonable scheduling it is very important.

Set network size is $\mathrm{N}$, the window size is $\mathrm{W}$, that is, the former $\mathrm{W}$ cells in each queue will compete the right to send the order. In fact, $\mathrm{W}$ is variable, then the optimization model as follows:

The objective function is $E=E_{1}+E_{2}+E_{3}$

Constraints conditions:

(1) All selected neurons have a different destination. This could be a selected $\mathrm{H}$ matrix, $H_{i j p q}=1$ indicates cell (i, j) and (p, q) have the same address. That calls conflict. Otherwise, $H_{i j p q}=0$, so

$$
E_{1}=\frac{A}{2} \sum_{i=1}^{N} \sum_{j=1}^{W} \sum_{p=1, p \neq i}^{N} \sum_{q=1}^{W} H_{i j p q} v_{i j} v_{p q}
$$

Here, only the selected cell is not blocked, $E_{1}=0$.

(2) Each window can only have one cell selected. Predetermined external input $I_{i j}=1$ indicates 
that their location has a cell, Otherwise, $I_{i j}=0$. Thus

$$
E_{2}=\frac{B}{2} \sum_{i=1}^{N} \sum_{j=1}^{W}\left(I_{i j}-\sum_{q=1}^{W} v_{i j}\right)^{2}
$$

Here, when all windows cell is selected, $E_{2}=0$.

(3) If cell has the higher position of the queue, it will have a greater priority and if the queue is longer , it will also have a greater priority. So

$$
E_{3}=-C \sum_{i=1}^{N} \sum_{j=1}^{W}\left(D-I_{i j}\right) v_{i j}
$$

\section{Conclusions}

In theory, the optimization algorithm designed based on Hopfield model: Neural network is stable, network will converge to the asymptotic equilibrium; Equilibrium point of neural network happens to be minimum point of energy function. In the actual operation process, the optimization algorithm based on the Hopfield model has the advantages of simple, normative and rapid. Optimization method of Hopfield model is not strict. The core strategy is gradient descent method. Therefore, sometimes the following problem inevitable occurred: Network convergence to local optimal solution; Network convergence to feasible solution; Robustness deviation problem caused by the improper selection of network parameters. Combining Hopfield model with competition network and making use of the competition network realize the constraint functions. The Hopfield neural network implementation has the advantage of simplifying the constraint of energy function. It will also greatly improve the algorithm performance; Combine Hopfield with GA (genetic algorithm) and a chaotic process, by using the ergodicity of chaotic dynamic and Advantages of parallel search of GA, it also can construct the effective optimization method.

\section{References}

[1] Xuefeng Zhao, Qin Ba, Lei Zhou, Weijie Li, Jinping Ou. BP neural network recognition algorithm for scour monitoring of subsea pipelines based on active thermometry. International Journal for Light and Electron Optics. Volume 125, Issue 18( 2014), p. 5426-5431.

[2] Hu Junguo, Zhou Guomo, Xu Xiaojun. Using an improved back propagation neural network to study spatial distribution of sunshine illumination from sensor network data. Ecological Modelling. Volume 266, Issue 24(2013): 86-96.

[3] Tuo zhong, Wang li-yuan. Improved BP Neural Network's Application in the Bank Early Warning. Procedia Engineering. Vol. 23 (2011), p. 216-221.

[4] Shiwei Yu, Kejun Zhu, Fengqin Diao. A dynamic all parameters adaptive BP neural networks model and its application on oil reservoir prediction. Applied Mathematics and Computation. Vol. 195 ( 2008), p. 66-75.

[5] Yi-Kuei Lin, Cheng-Ta Yeh. Multi-objective optimization for stochastic computer networks using NSGA-II and TOPSIS. European Journal of Operational Research. Vol. 218, Issue 3 (2012), p. 735-746.

[6] Yi-Kuei Lin, Cheng-Ta Yeh. Computer network reliability optimization under double-resource assignments subject to a transmission budget. Information Sciences. Vol. 181, Issue 3 (2011), p. 582-599. 
[7] Xiaoming Fu, Yang Chen, Guy Leduc, Laurent Mathy. Editorial for Computer Networks special issue on “ Measurement-based optimization of P2P networking and applications". Computer Networks. Vol. 56, Issue 3 (2012), p. 1077-1079. 